\title{
SMC: Uma Ferramenta Computacional para apoio ao Ensino dos Movimentos Circulares
}

\section{SMC: A Computational Tool for the Circular Movement Teaching}

\section{Carlos Vitor de Alencar Carvalho ${ }^{1}$ Janaina Veiga Carvalho ${ }^{2}$}

Artigo

Original

Original

Palavras-chave:

Resumo

Ensino de Física

Computação Gráfica

Sistema Computacional Educacional
Este trabalho apresenta o desenvolvimento do programa SMC para apoio ao ensino dos Movimentos Circulares Uniforme e Uniformemente Variado. É uma ferramenta de grande utilidade para professores e estudantes de Física, pois ela permite visualizar o sistema, modificar alguns dos seus parâmetros e estudar os seus comportamentos através de animações do movimento, ajudando bastante à compreensão do fenômeno físico.

\section{Abstract}

This work presents the development of the software SMC as a support to the teaching of the uniform circular movement and uniformly varied circular movement. It is a very useful tool for teachers and students of Physics, because it allows to visualize the system, to modify some of its parameters and to study its behavior through animation, helping the understanding of the physical phenomenon.
Key words:

Teaching of Physics

Computer Graphics

Educational Software 
O uso do computador para melhorar o processo ensino-aprendizagem já vem sendo discutido há algum tempo. A informática aplicada à educação tem sido utilizada de duas formas: para o próprio ensino da informática e para o desenvolvimento do ensino em outras áreas, como um recurso didático de apoio. Uma das formas desta utilização é o uso de programas computacionais para simular situações que, em determinados casos, só seriam possíveis de serem analisadas em experiências elaboradas em laboratórios.

No ensino da Física, esse aspecto é de grande importância, uma vez que além de simular diversos problemas físicos baseados em livros ou criados pelo professor/aluno, estes sistemas computacionais, ajudam no entendimento teórico de um sistema físico.

Um campo de ensino e pesquisa que tem evoluído nos últimos anos é a Computação Gráfica. Esse campo tem o objetivo de auxiliar na melhor percepção de conjunto de dados, descrevendo ambientes e visualizando processos de simulações por meios de suas técnicas (FOLEY et al, 1999). A Computação Gráfica pode atuar como mecanismo adicional para o desenvolvimento de programas computacionais educativos como, por exemplo, o uso de animações e gráficos, que podem tornar o conteúdo muito mais interessante.

O sistema SMC, que foi desenvolvido neste artigo, utiliza a Computação Gráfica como apoio para as visualizações. Eles foram desenvolvidos utilizando bibliotecas de código aberto e livre uso. Segundo Valente (1993), um programa computacional educativo, pode estar inserido em uma das seguintes categorias: Sistemas tutoriais, Sistemas de exercícios e práticas, Simulações e Jogos educacionais. Os sistemas desenvolvidos neste artigo estão inseridos no tipo simulações.

\section{Formulação Teórica e Desenvolvimento Computacional}

Nesta seção serão mostradas as formulações teóricas o Movimento Circular Uniforme e Uniformemente Variado, bem como o seu desenvolvimento computacional.
Os movimentos circulares utilizam expressões parecidas com as do Movimento Retilíneo Uniforme (COSTA et al, 2005) e Movimento Retilíneo Uniformemente Variável (SOBREIRA et al, 2006) com adaptações como velocidade angular ( ) e aceleração angular ( ). No movimento circular uniforme (MCU), a trajetória é circular e a velocidade escalar é constante não nula. A equação horária do espaço do MCU pode ser obtida dividindo ambos os membros da equação pelo raio $\mathrm{R}$ da trajetória circular. Logo, tem-se:

$\varphi=\varphi_{0}+\omega t$

Para o movimento MCUV, as equações horárias da velocidade e do espaço podem ser obtidas de forma similar. Logo, tem-se para a equação horária da velocidade:

$\omega=\omega_{0}+\gamma t$

e para a equação horária do espaço:

$\varphi=\varphi_{0}+\omega_{o} t+\frac{\gamma t^{2}}{2}$

Onde:

- $\varphi$ é a posição final;

- $\varphi_{0}$ é a posição inicial;

\subsection{Desenvolvimento Computacional}

A implementação das equações descritas acima foi feita em linguagem $\mathrm{C}$, utilizando o sistema de interface IUP (LEVY,1993) e o sistema gráfico OpenGL (WRIGHT et al.,1999). O OpenGL (Graphics Library) é uma interface para aplicações gráficas $2 \mathrm{D}$ e $3 \mathrm{D}$, independente do sistema de janelas e trabalha com primitivas geométricas e imagens. Possui uma arquitetura bem definida, boa performace, disponível em diversas plataformas e está bem documentado.

A interface desenvolvida pode ser visualizada na Figura 1. Nela, o usuário deve definir qual o movimento que será estudado (MCU ou MCUV) para dois objetos (pontos azul e vermelho) e visualizar os seus comportamentos através da uma animação gráfica. As funções horárias dos objetos são montadas a partir dos parâmetros definidos pelo usuário. 
Quando as posições de cada objeto são definidas, automaticamente as suas trajetórias circulares (em vermelho e azul na Figura 1) são desenhadas juntamente com a localização dos mesmos facilitando a interpretação do futuro movimento.

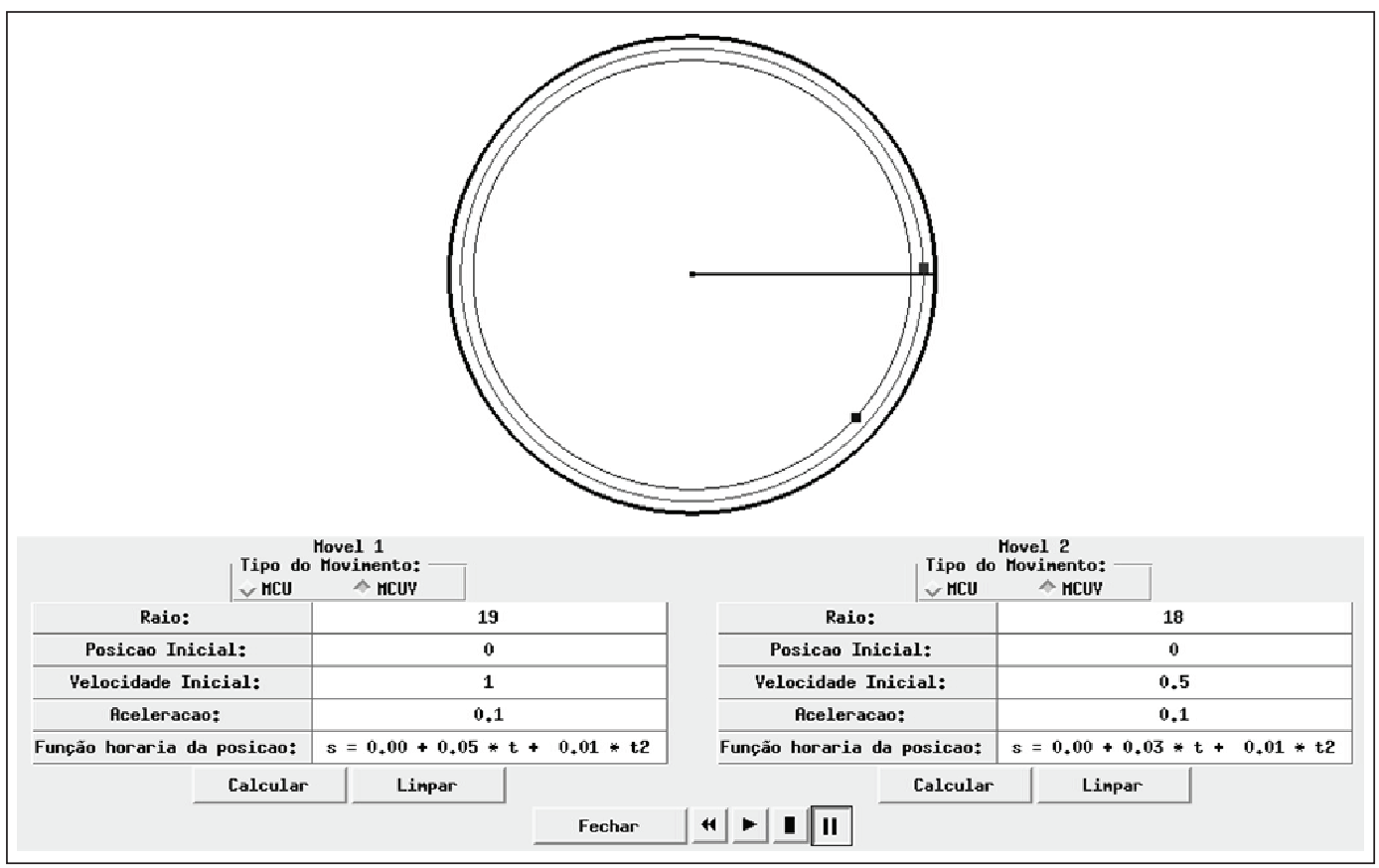

Figura 1 - Interface do módulo de Movimentos Circulares considerando um objeto em MCU e outro em MCUV.

As Figuras 2 mostram um exemplo da utilização do sistema. Nele são definidas as posições iniciais dos objetos e suas respectivas velocidades. Depois de uma volta na trajetória, podem-se verificar as funções horárias e verificar as novas posições dos objetos (Figura 3).

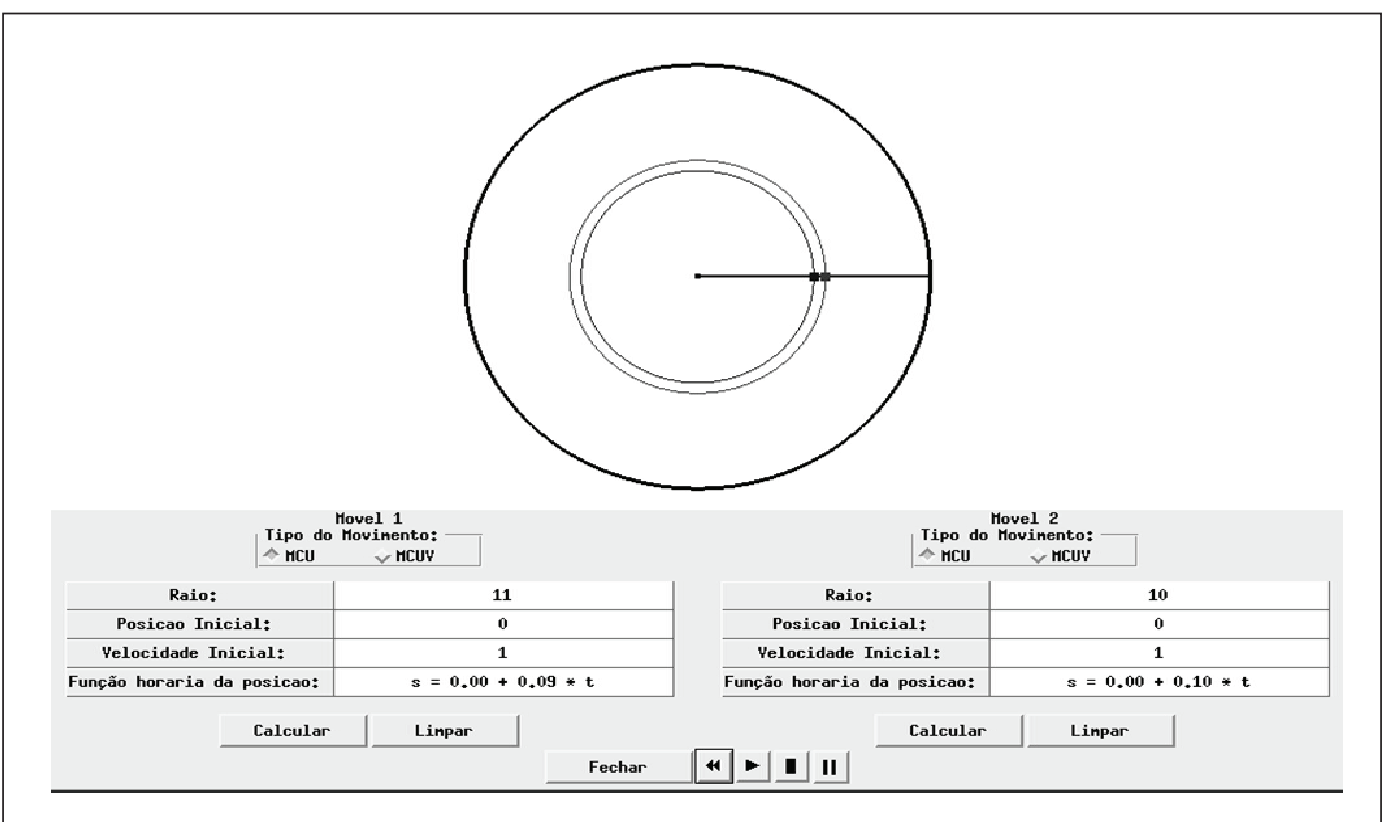

Figura 2 - Exemplo da aplicação do sistema mostrando as posições iniciais dos objetos. 


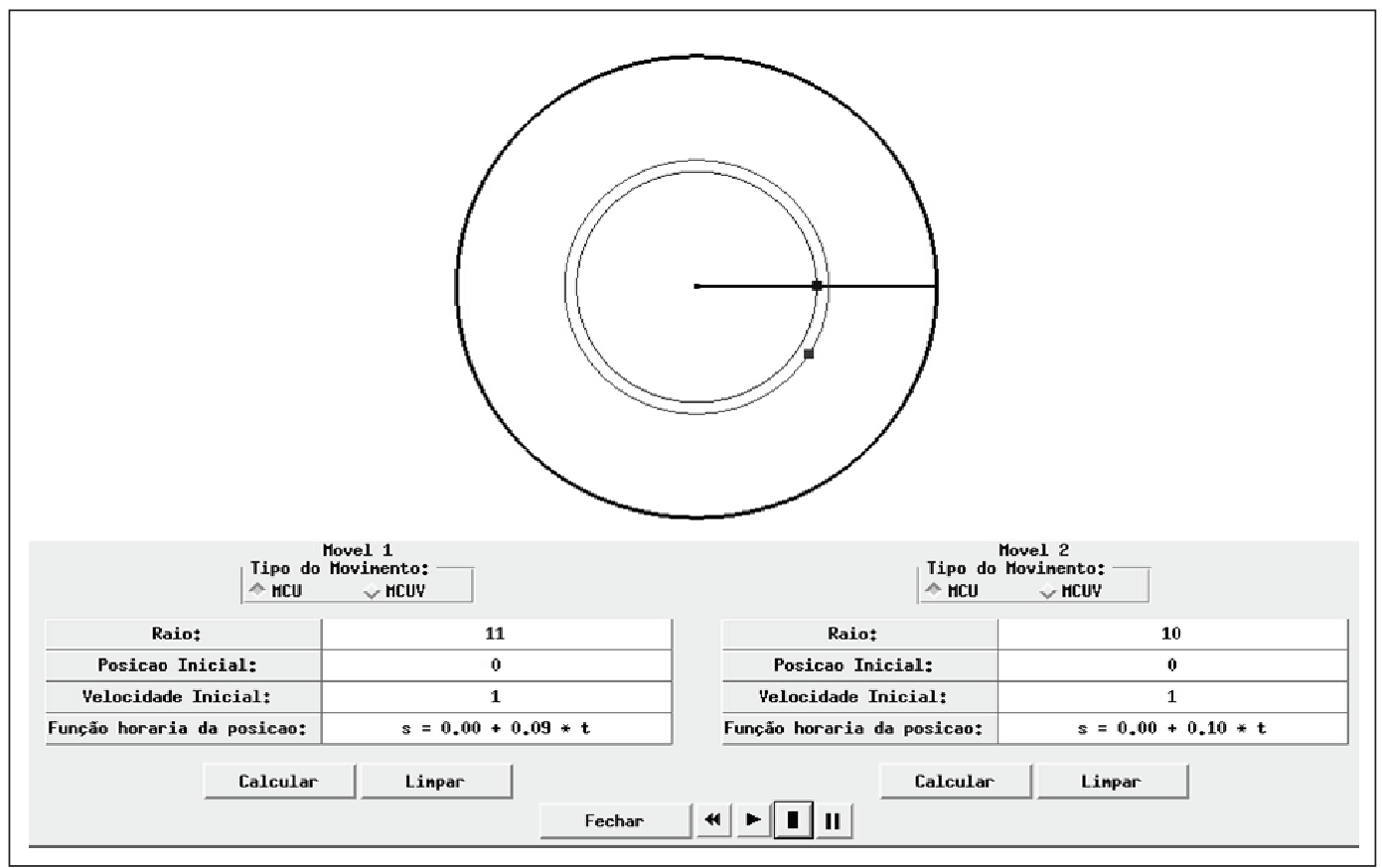

Figura 3 - Exemplo da aplicação do sistema mostrando as posições finais dos objetos depois de uma volta na trajetória.

\section{Considerações Finais}

O módulo descrito neste trabalho faz parte de um sistema mais amplo, composto de diversos outros módulos da Física, abrangendo as áreas da cinemática e dinâmica (CARVALHO et al 2007). A ferramenta desenvolvida, pode, como uma ferramenta complementar ao estudo tradicional, ajudar muito a compreensão dos fenômenos físicos envolvidos no sistema em questão. Com ela pode-se, em um curto espaço de tempo, fazer diferentes observações, contribuindo bastante para o entendimento da teoria abordada sobre o sistema em sala de aula. Porém, é importante ressaltar a integração com atividades desenvolvidas em sala de aula para que o aluno aumente o entendimento sobre o MCU e MCUV e a capacidade de resolver problemas desse tipo.

\section{Agradecimentos}

$\mathrm{O}$ primeiro autor agradece ao $\mathrm{CNPq}$ pelo apoio financeiro através da Bolsa de Produtividade em Desenvolvimento Tecnológico e Extensão Inovadora - DT.

\section{Referências Bibliográficas}

1. CARVALHO, Carlos Vitor de Alencar e Carvalho, Janaina Veiga Carvalho. Relatório Final do Projeto de Pesquisa : Pesquisa e Desenvolvimento de um Simulador e Interativo para Ensino da Cinemática e Dinâmica, Universidade Severino Sombra, 2007.

2. COSTA, Guilherme Cordilha Porto Coelho da; MENDES, Jorge Luís de Souza; CARVALHO, Janaina Veiga; CARVALHO, Carlos Vitor de Alencar.

Um sistema gráfico-Interativo para apoio ao ensino do movimento retilíneo uniforme. In: IV Encontro de Iniciação Científica e Pesquisadores da USS, Vassouras. IV Encontro de Iniciação Científica da USS. Rio de Janeiro : FUSVE, 2005. v. 1. . p. 1-3.

3. FOLEY, J.D. and VAN DAM, A. and FEINER, S. K. and HEGHES, J. F. Computer Graphics Principles and Practice, second edition in C, AddisonWesley publish company, 1999. 
4. LEVY, C. H., IUP/LED: Uma Ferramenta Portátil de Interface com

o Usuário. PUC-Rio, Rio de Janeiro1993 63f. Dissertação de Mestrado.

5. SOBREIRA, Guilherme; CARVALHO, Janaina Veiga; CARVALHO, Carlos Vitor de Alencar. MRUV: Um sistema computacional gráfico interativo para apoio ao ensino do Movimento Retilíneo Uniformemente Variado. In: V Encontro de Iniciação Científica e Pesquisadores da USS, Vassouras. V Encontro de Iniciação Científica e Pesquisadores da USS. Vassouras : FUSVE, 2006. v. 1. p. 1-3.

6. VAlENTE, J. A. Diferentes Usos do Computador na Educação. Em J.A. Valente (Org.), Computadores e Conhecimento: repensando a educação (pp.1-23). Campinas, SP: Gráfica da UNICAMP, 2003.

7. WRIGHT, R. S. Jr. and SWEET, M. OpenGL Super Bible, 2nd ed. Indianapolis, Indiana: Waite Group Press, 696 p, 2000.

Endereço para Correspondência:

Carlos Vitor de Alencar Carvalho cvitorc@gmail.com

Av. Expedicionário Oswaldo de Almeida Ramos, 280

Centro - Vassouras - RJ

CEP: 27.700-000

Informações bibliográficas:

Conforme a NBR 6023:2002 da Associação Brasileira de Normas Técnicas (ABNT), este texto científico publicado em periódico eletrônico deve ser citado da seguinte forma: CARVALHO, Carlos Vitor de Alencar; CARVALHO, Janaina Veiga. SMC: Uma Ferramenta Computacional para apoio ao Ensino dos Movimentos Circulares. Cadernos UniFOA. Volta Redonda, Ano V, n. 14, dezembro 2010. Disponivel em: <http://www.unifoa.edu.br/cadernos/edicao/14/25.pdf> 